\title{
Los intendentes en el gobierno de Guadalajara, 1790-1809
}

\author{
Carmen Castañeda \\ CIESAS de Occidente \\ Guadalajara, Jal., México
}

Estudio sobre la actividad de los intendentes de Guadalajara Jacobo Ugarte y Loyola, José Fernando de Abascal y Sousa y Roque Abarca en relación con las "causas de policía" de la ciudad del mismo nombre, en el virreinato novohispano. Se centra en los años anteriores al proceso independentista mexicano, después de aplicarse la Real Ordenanza de Intendentes de 1786. De acuerdo con ella, se dictaron normas —dadas a conocer a través de la Imprenta, que empezó sus actividades en esta ciudad en 1793- sobre la limpieza y reparación urbanas, nuevas construcciones e instituciones de servicio público, en conexión con las autoridades integrantes del Cabildo tapatío. Como conclusión interesante, el mantenimiento de la diferenciación social en la aplicación de esas medidas -abastecimiento de agua, empedrado de las calles-en beneficio más directo de la élite. El trabajo se basa en fondos documentales del Archivo General de Indias, la Biblioteca Pública del Estado y el Archivo Histórico Municipal, los dos últimos en Guadalajara, Jalisco.

\section{Presentación}

En 1992, con motivo de los 450 años de la fundación de Guadalajara, inicié la investigación de su historia urbana. Partí de lo que significó para la ciudad la primera división en cuarteles en $1790^{1}$ y el levantamiento del padrón militar en $1791 .^{2}$ Me interesaba averiguar cómo repercutía la división de su espacio y la cuantificación de sus habitantes en la urbe. Después estudié la política sanitaria de Guadalajara antes del establecimiento de las intendencias, ${ }^{3}$ así como también los cambios para mejorar la vida urbana, propuestos en 1790 por el primer intendente, don Antonio de Villaurrutia. ${ }^{4}$

1 Castañeda, Carmen: "Guadalajara hace 200 años: el reglamento de cuarteles de 1790 y el padrón de 1791", en Carmen Castañeda, coordinadora: Vivir en Guadalajara. La ciudad y sus funciones, Colección Guadalajara 450 años; 11, Guadalajara, 1992, págs. 41-57.

2 Castañeda, Carmen: "Una representación colectiva de Guadalajara en 1791" en The Urban History Worshop Review, vol. 3, Spring, 1996, págs. 1-9.

3 Castañeda, Carmen: "Vida material de Guadalajara: discurso, condiciones y propuestas para mejorar la sanidad de las calles, siglo XVIII", ponencia presentada en el II Coloquio de Historia Urbana, organizado por la Universidad de Guadalajara en Zapotlán el Grande, Jalisco, del 26 al 28 de febrero de 1998.

4 "Cambios para la vida urbana de Guadalajara en 1790", ponencia presentada en el Simposio "El impacto de las reformas borbónicas en la estructura de las ciudades, un enfoque comparativo", organizado por el Consejo del Centro Histórico de la Ciudad de México, del 22 al 24 de septiembre de 1999. 
Hoy quiero continuar con el examen del gobierno de los intendentes y su relación con la "causa de policía" en Guadalajara, ya que los intendentes tendrían que cumplir el artículo 68 de la Real Ordenanza para el establecimiento e instrucción de intendentes de exército y provincia. Estarían al tanto de que se cuidara la limpieza de los pueblos, ciudades y villas y del "ornato, igualdad y empedrados de las calles" y se vigilara que las construcciones no desfiguraran "el aspecto público", que las calles fueran "anchas y derechas y las plazuelas con la posible capacidad".

Esta encomienda aparentemente era sencilla. Pero, ¿cómo se podría lograr la limpieza y el ornato de Guadalajara, el empedrado y la alineación de las calles y la ampliación de las plazuelas? Precisamente, en esta ocasión voy a examinar la serie de "providencias gubernativas" o disposiciones para mejorar las condiciones urbanas de Guadalajara, sugeridas o expedidas por los intendentes, entre 1791 y 1809, y las implicaciones de tales medidas. Estos funcionarios entendían que sus obligaciones eran lograr "la quietud de los habitantes, seguridad de sus personas y propiedades, el beneficio público en todo lo posible y la recta administración de la justicia". Tareas que incumbían directamente al Cabildo de la ciudad, a los alcaldes ordinarios que se encargaban de las funciones judiciales y a los regidores que atendían las administrativas. La justicia y la administración eran "las dos grandes ramas de la gestión pública concejil"?

\section{Ugarte y las providencias de policía}

En 1791 el segundo intendente Jacobo Ugarte y Loyola tomó posesión de su cargo. A partir de ese año la vida empezaría a cambiar drásticamente en Guadalajara. La ciudad contaba, desde un año antes, con una división de su espacio en catorce cuarteles y con nuevas autoridades, los jueces mayores de

5 Real Ordenanza para el establecimiento e instrucción de intendentes de exército y provincia en el Reino de la Nueva España. 1786. Ed. Facsimilar. Introducción por Ricardo Rees Jones. México, 1984, fols. 78 y 79. Sobre este tema, véase Tau Anzoátegui, Víctor: Los bandos de buen gobierno de Buenos Aires en la época hispánica. Valladolid, 1983.

6 Boletín de la Sociedad Mexicana de Geografía y Estadística, 2. época, México, 1871, t. 3, págs. 316-320. Relación que forma [José Fernando Abascal], comandante general, presidente, gobernador e intendente de las provincias que comprende este reino de la Nueva Galicia; de las providencias expedidas desde el 10 de febrero de 1800, en que tomó posesión de estos empleos, hasta este día, con expresión de las resultas que han producido y remite a su Magestad, en cumplimiento de lo resuelto en real orden de 6 de mayo de 1792. Guadalajara, el 30 de septiembre de 1802.

7 Miranda, José: Las ideas y las instituciones políticas mexicanas. México, 1978, pág. 128. 
cuarteles y los alcaldes menores de barrios; las calles tenían, por primera vez, letreros con sus nombres en las esquinas y las casas sus números. Guadalajara experimentaría novedades mayores: se levantaría el primer censo moderno de su población (1791-1793); abrirían sus puertas la Real Universidad y el Colegio de San Juan (1792); empezaría a funcionar un taller de imprenta con su librería y el nuevo edificio del Hospital Real de San Miguel (1793); se establecería una comunicación periódica con la ciudad de México (1794) y se fundaría el Real Consulado de Comerciantes (1796).

Para poder llevar a cabo las "providencias" que tenía previstas, lo primero que hizo Ugarte y Loyola fue "formalizar" los reglamentos interinos de "los propios, arbitrios y bienes de comunidad", tanto de los pueblos de indios como de las villas y ciudades, entre éstas últimas, Guadalajara. ${ }^{8}$ De acuerdo con el reglamento los fondos de los propios de Guadalajara se componían de cinco ramos: el producto de sus ejidos (145 ranchos); el arrendamiento de las casas consistoriales; los censos a favor de la ciudad; el arrendamiento de "los puestos, cajones y jacales de los loceros" y el abasto de carnes. Los arbitrios de que disponía la ciudad provenían de tres ramos, venta de la plaza e impuesto de harinas y maíces. Los gastos que se hacían con los fondos de los propios y arbitrios eran de cuatro clases. De la primera se pagaba a los funcionarios municipales, de la segunda los réditos que reconocía la ciudad, de la tercera las "festividades votivas" y de la cuarta los "gastos fijos y eventuales".

Los reglamentos de propios y arbitrios como el de Guadalajara permitieron identificar los fondos disponibles, que ayudarían a resolver las necesidades de las poblaciones o a costear las obras que se emprendieran.

Después de arreglar lo correspondiente a los propios, arbitrios y bienes de comunidad, el intendente Ugarte y Loyola atendió los dos problemas principales de la ciudad, la necesidad que tenía de "empedrarse" para que sus habitantes se libraran de "un continuo denso polvo que produce no pocas fiebres éticas y tísicas y otros gravísimos perjuicios consiguientes a la desigualdad y barrancos que hay en algunas de sus calles, haciéndolas casi intransitables" y el problema de la escasez de agua que sufría la "numerosa población" de Guadalajara, "a pesar de los continuos crecidos gastos hechos para su introducción", ya que el vecindario apenas disponía

8 Archivo General de Indias (en adelante, AGI), Guadalajara, 358. "Reglamento interino formado para la administración, cuenta y razón de los propios y arbitrios que goza la ciudad de Guadalaxara en cumplimiento de los artículos 33 y 34 de la Real Ordenanza de Yntendentes de 4 de diziembre de $1786 . . ., 1792$. 
de "la precisa para no perecer, padeciendo algunas intercadencias que le ponen en las mayores angustias". 9

El intendente Ugarte pidió autorización al virrey para poder realizar las obras correspondientes. Antes fue necesario que el ingeniero de la ciudad de México, don Narciso Codina, levantase el plano de ella, "la nivelase y formase un presupuesto fiel del costo" del empedrado y que "reconociese las aguas de más segura permanencia, mejor calidad y menos costo, que levantase planos exactos y formase presupuestos útiles al fin". ${ }^{10}$

Ugarte y Loyola expresó que en esas dos "importantes obras de empedrar esta capital e introducirle con abundancia la agua de que carece", aunque fueron los objetos de su "mayor cuidado", nada se había adelantado entre 1791 y 1793, no obstante que el virrey, conde de Revillagigedo, había apoyado las iniciativas del intendente, porque la Junta Superior de Real Hacienda de México "acordó que del empedrado no se tratase hasta verificar la introducción de agua". ${ }^{11}$

Después de cuatro años Ugarte y Loyola pudo informar que gracias a la colaboración de "varios ciudadanos honrados, individuos algunos de este Ilustre Ayuntamiento", que ofrecieron "costear el empedrado de sus pertenencias o fincas", se pudieron empedrar las calles de las "casas de los ciudadanos pudientes y demás cuerpos políticos" así como las de las "fincas sujetas al fuero eclesiástico" porque el obispo don Juan Cruz Ruiz y Cabañas apoyó el proyecto. ${ }^{12}$

Desde 1745 el agua había llegado a catorce fuentes que se encontraban en los conventos (Santa María de Gracia, San Francisco, San Agustín, Santo Domingo, El Carmen, Jesús María, La Merced y Santa Mónica), en los colegios (Seminario Tridentino, San Juan, San Diego y Compañía de Jesús)

9 AGI, Guadalajara, 306. Boletín de la Sociedad Mexicana de Geografía y Estadística, t. 3, págs. 307-315. "Relación que forma [Jacobo Ugarte y Loyola], el comandante general, presidente, governador e intendente de las provincias que comprehende el reyno de la Nueva Galicia; de las providencias expedidas desde 14 de marzo de 1791, en que tomó posesión de estos empleos, hasta este día, con expresión de las resultas que han producido y remite a su Magestad por mano del Exmo. Sr. D. Pedro de Acuña, en cumplimiento de lo resuelto en real orden de 6 de mayo de 1792”. Guadalajara, 18 de diciembre de 1792.

10 Ibidem.

11 AGI, Guadalajara, 407. "Relación que forma [Jacobo Ugarte y Loyola] el comandante general, presidente, gobernador e intendente de las provincias que comprehende este reyno de la Nueva Galicia; de las providencias expedidas desde el 18 de diciembre de 1792 hasta este día, con expresión de las resultas y efectos que han producido las anteriores y remite a su Magestad por mano del Exmo. Sor. Dn. Pedro de Acuña, en cumplimiento de lo resuelto en real orden de 6 de mayo del año próximo pasado". Guadalajara, 18 de diciembre de 1793.

12 AGI, Estado, 43, núm. 28. "Edicto de Jacobo Ugarte y Loyola, gobernador e intendente de la Nueva Galicia, de dieciocho artículos con providencias sobre el empedrado”. Guadalajara, 1797, 12 págs. 
y en los hospitales de Belén y San Juan de Dios. También había fuentes en tres plazas (Mayor, de la Parroquia y de la Palma), el Real Palacio, la Cárcel, la Caja Real, la Real Aduana, el Cabildo y la Catedral. ${ }^{13}$ Junto a estas fuentes "públicas", sólo dieciocho personas dispusieron de agua en sus casas porque pudieron comprar una merced de agua a 500 pesos.

Todas estas fuentes sufrieron la escasez de agua en la última década del siglo XVIII, de ahí que Ugarte quisiera remediar los dos problemas, el del empedrado y el del agua. Pero aquí lo que interesa destacar es que el abastecimiento de agua a mediados del siglo XVIII y el empedrado de las calles, a fines, fueron dos obras de servicio público que marcaron las diferencias sociales y económicas de sus habitantes, ya que únicamente los ciudadanos honrados y pudientes y que podían disponer de 500 pesos pudieron tener agua en sus casas, la calle empedrada y así evitar el polvo.

Hira de Gortari ha destacado que en el cuerpo teórico de las reformas borbónicas estaban la doctrina de gobierno, que pretendía el control de los gobernados, y la doctrina de policía que aspiraba a mantener el orden y la seguridad de los ciudadanos. Mientras que el primer intendente ejemplificó perfectamente la doctrina de gobierno con "la creación de jueces mayores de cuarteles y alcaldes menores de barrios" y con la división del territorio de Guadalajara y sus barrios en catorce cuarteles, medidas que pretendían "la mayor cultura, quietud y civilidad de esta capital y los mayores auxilios de la justicia"; ${ }^{14}$ Ugarte siguió la doctrina de policía pues veló por la "paz y decoro interior" de los habitantes y promovió, "como parte esencial del buen orden", "la más exacta posible policía". En Guadalajara llegó a crear en 1795 la Junta, Juzgado o Tribunal de Policía "con el objeto único de procurar a este vecindario la mayor comodidad, y a las calles y edificios toda la hermosura y elegancia de que son susceptibles". ${ }^{15}$

Precisamente esta Junta de Policía le ayudó a organizar, con los "ciudadanos pudientes", el proyecto del empedrado y le sugirió que, para mantenerlo "con toda su firmeza y aseo", dictara "las providencias conducentes". Ugarte aprovechó la ocasión para recordar las ya publicadas en 1795 y prescribir otras que no tenían "otra mira ni otro interés que el bien general en la comodidad y salud pública". Reglamentó el tráfico de carretas que

13 AGI, Mapas y Planos, México, 153. Plano ignográfico de la ciudad de Guadalajara, 1745.

14 AGI, Guadalajara, 381. Biblioteca Pública del Estado en Guadalajara (en adelante, BPE), Archivo de la Real Audiencia de Guadalajara, Ramo Civil, expediente no catalogado. Ordenanza sobre "la creación de jueces mayores de cuarteles y alcaldes menores de barrios". Antonio Villaurrutia y Salcedo. Guadalajara, 4 de octubre de 1790.

15 AGI, Estado, 43, núm. 28. "Edicto de Jacobo Ugarte y Loyola", citado. 
transportaban "toda clase de materiales y víveres para su venta" o para su entrega, indicó los caminos que deberían seguir y señaló las plazas y lugares en donde tenían que depositar sus cargas. Dispuso el servicio de recolección de basuras por medio de un carretón y el sistema de multas para quienes las tiraran a la calle o en las esquinas.

Quizá la prohibición más drástica era la que tendía a suprimir aquellas actividades que regularmente se hacían en las calles: arrojar basuras, derramar aguas, sacudir ropas, "petates u otros efectos", asear coches, fregar utensilios, esquilar mulas y caballos, dejar carruajes sin mulas o bueyes y caballos afuera de las casas, tirar estiércoles o escombros, poner mercancías para su venta, y trabajar los "carrozeros, herradores, herreros, zapateros y cualesquiera otros menestrales".

Esta prohibición tuvo dos tipos de implicaciones, en primer lugar "la lógica de la calle" fue reconocida. ${ }^{16}$ Las personas podrían circular en los espacios públicos de las calles y tendrían que reducir al ámbito de sus casas las actividades que invadían las calles. La otra implicación tuvo que ver con la vida del trabajo, pues muchos oficios se ejercían en la calle, ${ }^{17}$ en donde la gente veía a los "menestrales", los reconocía con facilidad y los llamaba. Todos los que trabajaran en la vía pública o en el taller de su casa, que invadía los enlosados y las mismas calles, en ese "lugar intermedio entre el exterior y el interior", tendrían que dejar la calle libre. ¿Cómo reaccionarían los tapatíos ante la delimitación de los espacios de las calles para usos públicos y los de las casas para los privados?

Las calles serían objeto de más prácticas. La costumbre de regarlas y después barrerlas, todos los días en las mañanas muy temprano y en las tardes cuando estaba anocheciendo, que todavía seguían los tapatíos hasta hace unos años, fue establecida por el intendente Ugarte, quien señaló que "durante el tiempo de seca" se regarían "diariamente los frentes y costados de la pertenencia de cada vecino antes de la nueve de la mañana" y después se barrería "la basura gruesa que no sea tierra".

Otras medidas, relacionadas también con las calles, fueron expedidas por Ugarte, quien ordenó que sólo se vertiera el agua de los lavados "por los caños que de las casas caen por el suelo de las calles" y que se supri-

16 Sánchez de Tagle, Esteban: Los dueños de la calle. Una historia de la vía pública en la época colonial. México, 1997, pág. 102.

17 Para este asunto ver Arlette Farge: "Familias. El honor y el secreto", en La comunidad, el Estado y la familia, tomo VI, Philippe Ariès y Georges Duby (directores): Historia de la vida privada, Buenos Aires, 1991, págs. 188 y 189. Véase en especial el apartado "La calle y el taller". 
mieran los canales "en los altos de las casa" que arrojaban el agua a las calles y también las piletas o alcantarillas, que recogían "las aguas inmundas fuera de algunas casas". De estas tres medidas se empezaron a acatar la primera y la última, pero los tapatíos, hasta la fecha, han sido renuentes a cumplir la orden de retirar los canales o gárgolas de las azoteas.

En el gobierno del intendente Ugarte ya se habían concedido licencias para construir "portales con arcos", por lo que tuvo que prohibir que entraran coches, carretas y jinetes a los portales. A los cocheros les indicó que llevaran sus coches por el "centro de las calles, sin introducirse en los enlosados", ni "atropellando con insolencia al público". No correrían dentro de la ciudad y cederían el paso a los peatones.

Las providencias, expedidas por Ugarte, llegaron hasta tratar de "remediar la indecentísima costumbre que tiene la plebe de ambos sexos de ensuciarse en las calles y plazuelas". Los hombres que siguieran con esta costumbre serían destinados a las obras públicas y las mujeres a la "Teypa o Texqui”, como se le llamaba a la Casa de Recogidas. Los maestros de escuelas y los padres serían los responsables de los niños infractores y por tanto pagarían las multas.

Para elaborar toda esta serie de órdenes, Ugarte, probablemente, se basó en los "Apuntes de algunas providencias que exige la constitución de esta ciudad para que sea una de las más cómodas y sanas de la América", que tal vez preparó la Junta de Policía. ${ }^{18}$ Ugarte tomó en cuenta muchas de las providencias que se sugerían. Otras quedaron en el papel de los "Apuntes", tales como "matar todos los perros callejeros" y disminuir "los perros caseros"; vender los víveres "en sazón y bien condicionados"; mantener las casas, en que se congregara mucha gente, "bien aseadas, ventiladas y regadas"; establecer los hospitales "fuera del recinto del lugar"; "hacer letrinas públicas" con "separaciones para hombres y mujeres"; prohibir que se bañara la gente en las pilas públicas y "el uso de todo utensilio de cobre"; "disponer cementerios distantes de todo poblado" y "calles de árboles".

\section{Abascal y los problemas de la ciudad}

El 19 de agosto de 1798 murió el intendente Jacobo Ugarte y Loyola y el regente Francisco Saavedra y Carvajal se encargó del gobierno hasta el 10 de febrero de 1800, fecha en que el tercer intendente, José Fernando de

18 BPE, Fondos Especiales. Colección de Manuscritos. Ms. 14, 8 fols. 
Abascal y Sousa, tomó posesión de su cargo. ${ }^{19}$ Aunque Abascal atendió los asuntos de toda la provincia — bandidos, delincuentes, tributos, alcabalas, azogues, educación, puentes y caminos- puso especial atención a los problemas de la ciudad de Guadalajara.

Inmediatamente descubrió y persiguió "el vicio del juego, tan dominante en América, que con ruina lastimosa de un sinnúmero de familias, estaba escandalosamente radicado en esta capital". Con las "multas exigidas a viciosos en juegos prohibidos" y una parte de lo producido en las corridas de toros pudo emprender obras en beneficio de los tapatíos, la continuación del empedrado y un paseo "de 536 varas ... entre los puentes de San Juan de Dios y el de Medrano".

Aunque Ugarte había declarado que 1797 podía considerarse el año más feliz de Guadalajara porque el gobierno había resuelto los dos principales problemas de la ciudad, el empedrado y la introducción del agua dulce, que empezaba "a correr a satisfacción del público con el aumento de quatro fuentes nuevas", que se estaban construyendo, el intendente Abascal no corroboró sus buenas intenciones y en septiembre de 1802 informó al rey que no se había podido conseguir "la provisión de agua abundante, como precisa, para el uso de toda clase de manufacturas", que su antecesor había abandonado la obra del empedrado "por impracticable", y que él sí había vencido las dificultades y "sin el menor tropiezo ni oposición" se hicieron en año y medio "98,000 varas cuadradas" de empedrado, que con las " 86,000 que ya lo estaban", sólo faltaría una cuarta parte para terminarla con solidez y hermosura, una empresa tan útil como deseada. El costo del empedrado lo habían pagado "con mucho gusto los dueños de fincas, pudientes" más "un fondo" que dio el obispo Cabañas y "el resto producido de multas y corridas de toros". ${ }^{20}$

El paseo público se construyó en un terreno que "era el recipiente de todas las inmundicias de la ciudad, cuya parte inmediata a tal pestilencia, padecía considerablemente en la salud de sus vecinos". Para Abascal, "el delicioso paseo" se convirtió en "un desahogo inocente de que carecía esta considerable población", que ayudaría a distraerla "de las concurrencias privadas y por lo regular perniciosas". Para el financiamiento de esta obra, "adornada de cuatro filas de árboles, muchos asientos cómodos de cante-

19 Gálvez Ruiz, María Ángeles: La conciencia regional en Guadalajara y el gobierno de los intendentes (1786-1800). Guadalajara, 1996, pág. 31.

20 Boletín de la Sociedad Mexicana de Geografía y Estadística, t. 3, págs. 316-320. Relación que forma [José Fernando Abascal]. Guadalajara, el 30 de septiembre de 1802. 
ría, pilastras y columnas de buen gusto", se volvió a contar con "una suscripción voluntaria de los cuerpos y vecinos pudientes de la ciudad".

Además del empedrado y del paseo, Abascal mandó "construir unos baños públicos, cómodos, sanos y decentes" en el barrio de Mexicaltzingo, pues se había dado cuenta que en el tiempo de secas que dura "ocho meses del año que deja de llover", la gente buscaba "su refrigerio en cualquier charco, por cenagoso o puerco" que fuera y "sin el rubor de ser vista en cueros". Para el intendente esta costumbre producía "funestas consecuencias", que lo obligaron a "buscar arbitrios" para la construcción de los baños. Pudo emprender la obra con los fondos que había para la reedificación del templo de Mexicalztingo, un "poco mas de cuatro mil pesos", que se tomaron de acuerdo con el obispo Cabañas, el párroco y los parroquianos de Mexicaltzingo. Con esta cantidad más "una limosna de mil y más pesos", que ofreció el obispo, Abascal pudo concluir los baños, que tuvieron una "sencilla y arreglada arquitectura de la galería", formada por "quince arcos de cantería", sin contar "el aseo y decencia de los aposentos y buena forma de las pilas".

Con "el considerable rédito" que producía "el caudal invertido" se pudo hacer la reconstrucción del templo y después quedó "a beneficio del mayor culto de aquella milagrosa imagen del Santísimo Cristo de la Penitencia", que se veneraba en la iglesia parroquial de Mexicaltzingo.

Al intendente Abascal también le correspondió la construcción de un parián en la plaza de San Agustín, ya que el "voraz incendio acontecido el 31 de mayo de 1795" había destruido "los cajones o tendejones de madera, en que se vendían comestibles en esta plaza mayor, y en cuyo desgraciado accidente estuvieron muy a riesgo de perderse los crecidos caudales del comercio" que había en las casas inmediatas. Con la aprobación de la Junta Superior de Real Hacienda dispuso de casi siete mil pesos de los propios de la ciudad para esa obra que benefició al público, "vendedores y compradores de toda clase de comestibles", y que además obtenía "un rédito de más de un doce por ciento".

\section{Abarca y las providencias de fácil observancia}

Abascal dejó el gobierno de la Intendencia de Guadalajara el 22 de septiembre de 1804 y fue sustituido por el regente José Ignacio Ortiz de Salinas, cuya gestión duró siete meses pues el 2 de mayo de 1805 llegó a Guadalajara el nuevo intendente, Roque Abarca, quien atendió tanto la cau- 
sa de gobierno como la de policía. Como sus antecesores, también pensó en expedir las providencias que fueran "de fácil observancia al público", que consultó con la Real Audiencia. Este tribunal le recomendó formar un nuevo "establecimiento de cuarteles", pues era el medio "más propio para asegurar la tranquilidad pública y facilitar a la Junta de Policía los auxilios que necesita para llenar los objetos de su instituto". ${ }^{21}$

Por medio de un bando, Abarca dio a conocer la división de la ciudad en veinticuatro cuarteles y los nombres de los alcaldes de cada cuartel, "sus funciones y las obligaciones del vecindario". Si comparamos esta nueva división con la primera de 1790 veremos que el territorio de los cuarteles fue más pequeño en la segunda división para lograr un mayor control de los vecinos. Esta división se mantuvo por lo menos unos trece años para la policía de la ciudad y los padrones que se levantaron..$^{22}$

Igualmente, Abarca mandó publicar un bando para "hacer entender a el público las disposiciones relativas a la Policía, en los términos pedidos por la Junta que aprobó la Real Audiencia”. En primer lugar estaban las dos disposiciones sobre la Junta de Policía y sus subalternos y después las treinta que debería observar el público. De acuerdo con estas disposiciones, la Junta de Policía estaría presidida por el intendente y dos regidores y debería de "fomentar el ornato, aseo y limpieza de la población en sus edificios y en sus calles, dictar las providencias oportunas al logro de estos recomendables objetos que tanto influyen en la salud pública".

El intendente Abarca estaba convencido que los tapatíos se habían olvidado de todas las providencias expedidas anteriormente sobre la policía y el buen gobierno de la ciudad, por lo que decidió renovar, en 1809, "los bandos publicados a este efecto". Por esta razón casi no encontramos novedades en las disposiciones que pretendían mantener limpia Guadalajara y conservar en buen estado el empedrado: regar las calles; quitar las piletas de las vías públicas y construirlas en el interior de las fincas; suprimir los canales de aguas sucias; no arrojar las basuras en las calles; echar otras basuras (cisco, estiércol, zacate, hierbas, animales muertos) en los sitios destinados por la Junta; situar a los herradores en otros lugares que no fueran las calles; no llevar bestias, carruajes ni carretillas a los por-

21 Archivo Histórico Municipal de Guadalajara. "Disposiciones relativas a la Junta de Policía y sus subalternos y disposiciones que debe observar el público", de Roque Abarca. Guadalajara, 19 de agosto de 1809 .

22 Para la división de la ciudad en veinticuatro cuarteles y los padrones de 1821 y 1822 ver: Anderson, Rodney D.: Guadalajara a la consumación de la Independencia: estudio de su población según los padrones de 1821-1822. Traducción de Marco A. Silva. Guadalajara, 1983. 179 pág. 
tales; no correr carruajes por las calles; no dejar sueltas las mulas; no ensuciarse en las zonas públicas y hacer letrinas en las casas; no hacer hoyos ni agujeros en las calles sin licencia de la Junta; no arrastrar maderas ni otra cosa que pudiera descomponer el empedrado. Con estas medidas, se recordaba a los habitantes de Guadalajara el carácter público de las calles.

Tanto Ugarte como Abascal habían acatado la primera parte del artículo 68 de la Real Ordenanza para el establecimiento e instrucción de intendentes de exército y provincia, pues habían estado al tanto de cuidar la limpieza de Guadalajara y del "ornato, igualdad y empedrados de las calles". Al intendente Abarca le correspondió vigilar que las construcciones no desfiguraran "el aspecto público" y que las calles fueran "anchas y derechas y las plazuelas con la posible capacidad". ${ }^{23}$ Para conseguir esto ordenó que no se hicieran "fábricas sin licencia de la Junta", es decir, que no se construyera "casa chica o grande, jacal, cerca de solar" sin previo permiso. Igualmente se necesitaría licencia para "ocupar la calle con escombros". Prohibió que se edificaran casas "sin guardar la línea de las fabricadas en el centro" o que se pusieran "repisas de ventanas bajas voladas". Obligó a los dueños de "casas ruinosas" a repararlas y que cuando se hicieran casas nuevas o se derribaran las antiguas quedaran "las calles anchas y derechas y las plazuelas con la posible capacidad". Todas estas órdenes y prohibiciones repercutirían en el ordenamiento urbano.

Abascal se distinguió de Ugarte y de Abarca porque en su informe mencionó las funciones propias de su cargo de gobernador e intendente, entre ellas "el despacho diario de los muchos negocios de todos los ramos de gobierno y real hacienda". Seguramente que los tres atendieron los asuntos de la Intendencia aunque destacaron todas las medidas que aplicaron para la policía y el gobierno de Guadalajara. Da la impresión de que entre 1791 y 1809 ellos gobernaban la ciudad, sin embargo, la creación de la Junta de Policía por Ugarte y respaldada por Abascal y Abarca permitió la relación con el Ayuntamiento a través de los regidores, encargados de la regulación y el buen funcionamiento de la vida urbana. Era responsabilidad de estos funcionarios el aprovisionamiento de la población, la adjudicación de tierras, la vigilancia de los propios y ejidos, el ordenamiento de las actividades de los artesanos, la elaboración de estatutos de los gremios, la fijación de los precios y muchos otros asuntos, como reglamentar las condiciones de sanidad, recaudar los propios de la ciudad,

23 Real Ordenanza para el establecimiento e instrucción de intendentes..., fols. 78-79. 
pagar los salarios a los funcionarios y, en pocas palabras, de todas "las incumbencias de la república". ${ }^{24}$

\section{La cultura impresa y el gobierno urbano}

Los tres intendentes recurrieron a la cultura impresa para difundir las "providencias gubernativas" que mejorarían las condiciones urbanas de Guadalajara. Los tres publicaron bandos impresos con las medidas que debía observar la gente y las multas para aquéllos que no cumplieran las disposiciones. Con los bandos colocados en los edificios públicos y leídos para todo el mundo nadie alegaría ignorancia.

Antes de 1810 la cultura impresa ganó terrenos en Guadalajara, no tanto con los libros sino con los periódicos. Los tapatíos también leían publicaciones periódicas. Unos cuantos estaban suscritos a la Gazeta de México y al Diario de México, otros recibían la Gazeta de Madrid y los comerciantes del Real Consulado leían el Semanario de Agricultura y Artes. Dos periódicos españoles se leyeron con mucho interés en 1808, tanto que las autoridades ordenaron la reimpresión en Guadalajara del Correo Político y Literario de Salamanca y del Semanario Patriótico, publicado primero en Madrid, luego en Sevilla y por último en Cádiz. ${ }^{25}$

La causa de policía también se aprovechó del medio impreso que representaba el periódico, pues el miércoles 13 de diciembre aparecieron publicadas, en el Diario de México, nueve "Providencias de policía que hacen falta en esta ciudad de Guadalaxara" ${ }^{26}$ La primera era la falta de "iluminación de sus calles por la noche, con la custodia de los guardias, que llaman serenos". Le seguía la necesidad de "contener el progreso de las barrancas, que por todas partes van circulando a esta ciudad, dificultando sus salidas". En tercer lugar se recordaba que estaba prohibido que en la plaza mayor, en los portales y en las banquetas o aceras de las calles, entraran "carruajes, carretillas y toda bestia de carga o de tiro". Luego venía la sugerencia de plantar y colocar "en simetría, algunos árboles de los mas

24 Mota Padilla, Matías de la: Historia del reino de Nueva Galicia en la América Septentrional. Guadalajara, 1973, cap. XCIII, pág. 505.

25 Castañeda, Carmen: Imprenta, impresores y periódicos en Guadalajara, 1793-1811. Guadalajara, 1999.

26 "Providencias de policía que hacen falta en esta ciudad de Guadalaxara", en Diario de México, núm. 1534, t. XI, pág. 673, miércoles, 13 de diciembre de 1809. 
copudos, como los fresnos" en la plaza mayor "para su mayor adorno y comodidad". También se pedía que las casas, que se construyeran o renovaran, tuvieran "embutidas en la pared los canales de las azoteas", como estaba ordenado en la ciudad de México, y que las "rejas y ventanas baxas", que daban a la calle, deberían "colocarse de modo que no sobresaliesen de la pared" y "ponerse todas a altura suficiente, para que el hombre de más talla pudiese pasar por baxo de ellas, sin doblarse".

No faltaron las providencias sobre las calles. Se pedía que hubiera "una permanente disposición del gobierno, depositada en el ayuntamiento, relativa a la altura, anchura y declive", que deberían tener las banquetas y que el declive que se dejara "en lo ancho de las calles, desde las paredes hacia el medio, así en los empedrados, como en los terraplanes", debería ser "muy tenue e insensible y absolutamente ninguno en la anchura de las banquetas".

Para remediar las desigualdades en las calles no empedradas de esta ciudad, se proponía que se empleara a "algunos forzados" para que rebajaran "la tierra de las partes elevadas de dichas calles, para acomodarla, bien pisoneada en las partes baxas inmediatas", de esta manera todo el suelo quedaría plano. El empleo de los presos para las obras públicas era una medida que había sido propuesta por Abarca "para auxiliar al asentista de la limpia de las calles" y para que "con más presteza y facilidad" se pudiera realizar "el terraplén de las calles u otro reparo urgente" que determinara la Junta. Para esto los juzgados ordinarios de la ciudad facilitarían a la Junta "todos los presos de delitos leves que les pidieren".

\section{Conclusiones}

La revisión de las providencias expedidas por tres intendentes en relación con la "causa de policía" en Guadalajara ha mostrado que atendieron primero la limpieza de la ciudad y después vigilaron que las construcciones no desfiguraran "el aspecto público", tal como lo pedía el artículo 68 de la Real Ordenanza para el establecimiento e instrucción de intendentes de exército y provincia. Los tres reiteraron a la población tapatía el cumplimiento de las órdenes. Aunque esta insistencia en las mismas disposiciones indica que no se acataban, las providencias expedidas sí lograron producir costumbres, como sería barrer las calles o recoger la basura, que serían las que prevalecerían. 
Las medidas tomadas por los tres intendentes en Guadalajara redundaron en una transformación del espacio urbano al otorgarles a las calles su carácter público y reservar para las casas las actividades de tipo privado. Estos cambios se basaron en la división del espacio citadino en cuarteles y su vigilancia por los alcaldes de barrio. Además, con el gobierno de los intendentes la ciudad contaría con el primer paseo y los primeros baños, obras de servicio público que se opondrían a las de carácter privado supuestamente perniciosas, mientras que las primeras, al estar a la vista de todos, serían vigiladas.

Los intendentes, a través de la Junta de Policía, pudieron mantener la relación con el Cabildo de la ciudad, integrado por los alcaldes ordinarios que se encargaban de las funciones judiciales y los regidores que atendían las administrativas. Ambos, alcaldes y regidores, pertenecían a la élite de Guadalajara, constituida por hacendados y comerciantes.

Los tres intendentes se involucraron con los dos mayores problemas que tenía la ciudad, el abastecimiento de agua y el empedrado de sus calles. Las medidas para resolverlos fueron prácticas de diferenciación social, ya que únicamente los ciudadanos pudientes dispusieron de agua en sus casas y de calles empedradas; el resto de la población tuvo que conformarse con obtener el agua en las fuentes públicas y con sufrir el polvo por la falta de empedrado.

Entre los múltiples usos de la imprenta, que había llegado a Guadalajara en 1793, también estuvo la publicación de las providencias de policía que colaboraron al incremento de la cultura impresa en la ciudad. 